\title{
SINGULAR PERTURBATIONS FOR THE FORCED VAN DER POL OSCILLATOR
}

\author{
E. M. ELABBASY
}

\begin{abstract}
This paper deals with the Van der Pol oscillator with large sinusoidal forcing term. By using singular perturbation techniques asy mptotic solutions of such a system are constructed. We considered the case $b$ small and then we find the range of values of $b$ for which the Van der Pol oscillatore may have 3 stable subharmonic solutions for the same values of the parameters. We have integrated the equation numerically; then a comparison of the numerical results obtained with analytical results of this paper is given.
\end{abstract}

\section{Introduction}

We consider the forced Van der Pol oscillator

$$
\frac{d^{2} x}{d t^{2}}+k\left(x^{2}-1\right) \frac{d x}{d t}+x=k b \lambda \cos \lambda t
$$

where $b, \lambda$ and $k$ are parameters independent of $x, \frac{d x}{d t}, t$ and $b, \lambda$ independent of $k$. This is a prototype for second order ordinary differential equations in which the coefficient of the derivative of the dependent variable changes sign-corresponding physically to damping which changes sign.

For $b=0$, equation (1.1) is an autonomous and the behaviour of the solutions are well known. It has been a subject of investigation both analytically and numerically for many years ago. Among the most important numerical result of the periodic solutions of the autonomous Van der Pol's equation is as follows:

Received May 15, 1992; revised October 8, 1992. 
When $k$ increases from 0 , the amplitude $a$ of the limit cycle (periodic solution) of the Van der Pol Oscillator increases monotonically from 2 unitl $k=3$ and decreases monotonically after $k=4$. The maximum of the amplitude is attained at $k=3.265$ and its value is $a_{\max }=2.035$ (c.f [3] and [8] fordetails). This conclusion is quite interesting because such a minute change of the amplitude could hardly be determined by any graphical solution or analog computation.

The amplitude $T_{0}$ of the limit cycle of the Van der Pol oscillator is an increasing function of $k$. An asymptotic expansion for the periodic solution of the autonomous Van der Pol equation with $k \gg 1$ has been given by Dorodnicyn [2] and Cole and Kevorkien [5]. In [8] Urable corrects the higher terms in Dorodnicyn's formula for the period. It would be possible to find more details of the expansion for the period of the limit cycle. For $k \gg 1$, the period $T_{0}$ of the limit cycle is approximated by

$$
T_{0}(k)=(3-2 \ln 2) k+O\left(k^{\frac{-1}{3}}\right),
$$

For $b>0$ the situation is quite different. The equation may have a periodic solution with a period $m$ times the period of the forcing term, i.e., of period $m 2 \pi / \lambda$. This phenomenon is called harmonic entrainment when $m=1$. Higher harmonic entrainment occurs when $m=\frac{1}{p}$ for some integer $p$. In this paper we investigate only the third kind of synchronization, that is subharmonic entrainment, leading to periodic solutions with period $m 2 \pi / \lambda$ where $m>1$. Such a subharmonic solutions arise when the period $T_{0}$ of the autonomous equation is sufficiently close to $2 m \pi / \lambda$.

In [6], [7] Littlewood studied this equation in great details when $b<2 / 3$ and $k$ large or more accurately, when $k$ exceeds some $k_{0}(b, \lambda)$. It has been established that 0 and $2 / 3$ are the only critical values of $b$ and that the behaviour for $0<b<2 / 3$ is excedingly complicated. The interval $(0,2 / 3)$ consists of three disjoint sets: a set $S_{1}$ of intervals $I_{1}$, another set $S_{2}$ of intervals $I_{2}$, and very short transitional set $S_{3}$ of intervals $I_{3}$ between $I_{1}$ and $I_{2}$. When $b \in S_{1}$, there is just one stable subharmonic solution of order $2 n+1$ (i.c. of period $(2 n+1) 2 \pi / \lambda$ ), when $b \in S_{2}$ there are two stable subharmonics of orders $2 n+1$ and $2 n-1$. 
As $b$ increases through $I_{1}$ and $I_{2}$ (jumping the transitional intervals $I_{3}$ ) there are successively one subharmonic of order $2 n+1$; two subharmonics of orders $2 n+1,2 n-1$; one subharmonic of order $2 n-1$; two subharmonics or orders $2 n-1,2 n-3$; and so on. $n$ is of the order $(2 / 3-b) k$. However, this formula for $n$ was proved to be incorrect, in [4] a modified formula for $n$ has been given and was found to be $n=(2 / 3-b) k / 3$. When $b \in S_{3}$ the situation in this case may be very difficult to predict. However, as $b$ crosses the gap $I_{3}$, a complicated sequence of bifurcation may occur. For some values of $b$ periodic stable points may exist. Despite their stability, it would be very difficult to detect them on the computer due to their high period and small basin of attraction. For $b>2 / 3$ equation (1.1) has only one stable periodic solution of period $2 \pi / \lambda$.

\section{General Theory and Global Properties}

2.1 General Theory. We seek periodic solutions of equations (1.1) $(b \neq$ $0)$. If $x(t)$ is a solution of (1.1) with period $T$, we have $x^{m}(t)=x^{m}(t+T)$, $m=0,1,2, \cdots$, so that equation (1.1) gives $\sin \lambda t=\sin \lambda(t+T)$ for all $t$. Hence all periodic solutions of equation (1.1) have period $2 m \pi / \lambda$, for some integer $m$. When $m$ is odd this periodic solution is called normal subharmonic, while if $m$ is even the solution is called abnormal subharmonic.

From the general theory (c.f. [1], Theorem 1 ) every trajectory of equation (1.1) eventually satisfies

$$
|x(t)|<A,|\dot{x}(t)|<A(k+1),
$$

where $\dot{x}=\frac{d x}{d t}$ and $A$ is constant independent of $k$.

2.2 Global Properties. The curve $y=F(x)=\frac{x^{3}}{3}-x=\int_{0}^{x}\left(s^{2}-1\right) d s$, intersects the line $y=b$ at three different points if and only if $\frac{(3 b)^{2}}{4}+\frac{(-3)^{3}}{27}<0$; that is $b<2 / 3$. This is precisely the condition of the problem.

Lemma 1. A maximum can occur only when

$$
\frac{\pi}{2 \lambda}+\frac{2 m \pi}{\lambda}-\frac{A k^{-1}}{\lambda}<t<\frac{3 \pi}{2 \lambda}+\frac{2 m \pi}{\lambda}+\frac{A k^{-1}}{\lambda}
$$


and a minimum only when

$$
\frac{3 \pi}{2 \lambda}+\frac{2 m \pi}{\lambda}-\frac{A k^{-1}}{\lambda}<t<\frac{5 \pi}{2 \lambda}+\frac{2 m \pi}{\lambda}+\frac{A k^{-1}}{\lambda} .
$$

Proof. First we show that $\ddot{x} \neq 0$ at an extremum. If $\ddot{x}=0$ were possible, then from equation (1.1)

$$
x(t)=k b \lambda \cos \lambda t
$$

Now differentiating equation (1.1) with respect to $t$ at such point would give $x=-k b \lambda^{2} \sin \lambda t$. But it is necessary for an extremum that $x=0$, so that $\sin \lambda t=0$. Hence, from (2.2.1) we would have $|x(t)|=k b \lambda$, a contradiction to boundedness condition (2.1.1).

Therefore $\ddot{x} \neq 0$ at an extremum. Now at a maximum $(\dot{x}=0, \ddot{x}<0)$, we have from equation (1.1) that $\cos \lambda t<x / k b \lambda$, and at a minimum $(\dot{x}=0, \ddot{x}>0)$, $\cos \lambda t>x / k b \lambda$. These inequalities give the result.

Lemma 2. All the periodic solutions of equation (1.1) are oscillating.

Proof. We prove that all periodic solutions of equation (1.1) are oscillating in the sense that there is a montonic sequence $t_{n}$ such that $t_{n} \rightarrow \infty$ as $n \rightarrow \infty$ and $x\left(t_{n}\right)=0$. Assume the contarary that there is $\propto$ such that $x(t)>0$ for all $t>\propto$. From lemma 1 we see that the trajectory of (1.1) lies in $1 \leq x \leq 2$ for $\left(2 m+\frac{1}{2}\right) \pi / \lambda \leq t \leq\left(2 m+\frac{3}{2}\right) \pi / \lambda$. Suppose that after the first such point in this interval, the trajectory remains in $x \geq 1$ for a time $\Theta_{m}$ while $t<(2 m+2) \pi / \lambda$. If $B$ is chosen so that $|\dot{x}| \leq B K$ for $t>\propto$, it follows that either

$$
1=\int_{x=1}^{x=2} x^{-}(t) d t<B K \Theta_{m}
$$

or $x>1$ until $t=(2 m+2) \pi / \lambda$. In both cases we have

$$
\int_{t=2 m \pi / \lambda}^{t=(2 m \pi+2) / \lambda} x(t) d t>A k^{-1}
$$

Now integrating equation (1), we get

$$
(X(t)+\dot{x}) k^{-1}=-F(x)+b \sin \lambda t+C,
$$


where $X(t)=\int_{t_{0}}^{t} x(s) d s, F(x)=x^{3} / 3-x$ and $C$ is the constant of integration.

Hence, (2.2.1) implies that $X(t) \rightarrow \infty$ as $t \rightarrow \infty$ and then $F(x)=b \sin \lambda t+$ $C-\dot{x} k^{-1}-X k^{-1} \rightarrow-\infty$ as $t \rightarrow \infty$. So that $x(t)$ becomes negative, a contradiction to the assumption $x(t)>0$ for all $t>\propto$. This completes the proof of the Lemma 2.

The trajectory of a stable subharmonic solution of order $2 n \pm 1$ is skew symmetric since $x(t)=-x\left(t+\left(n \pm \frac{1}{2}\right) 2 \pi / \lambda\right)$. It has an $(n \pm 1 / 2)$ of waves above the $t$-axis, and intersects the $t$-axis twice in the period. The upper set of waves can (for part of the range of $b$ ) make a few dips (none for a certain range of b), with depth of order $k^{-\frac{1}{2}}$ below $x=1$. This phenomenon is known as the depth phenomenon and it has been discussed in details in [6]. The neighborhood of $x=1$, where the damping changes sign is highly critical. For $|X|<1$ the damping is negative and then it increases the amplitude of of the oscillation. The trajectory crosses this interval in a time o(1). A schematic graph of the trajectory of a stable normal subharmonic of equation (1.1) is given in Fig. 1.

\section{Littlewoods's problem}

The so called Littlewood's problem; problem 29 [7] for the Vander Pol's equation (1.1) consists of a number of unsolved problems. Among these unsolved problems is the case when $b=\epsilon_{k}$, where $\epsilon_{k}$ is small for large $k$, e.g. $\epsilon_{k}=k^{-a}$ for some positive number $a$. Another interesting, and probably the most difficult unsolved problem, is the case equation (1.1) has 3 stable subharmonic solutions for the same value of the parameters $k, b$ and $\lambda$. Although this problem has been stated by Littlewood [7] twenty five years ago, nothing has been published previously.

In this paper we consider these two problems. We take $b=\beta k^{-1}$, where $\beta$ is a positive constant independent of $k$. Equation (1.1) then becomes

$$
\frac{d^{2} x}{d t^{2}}+k\left(x^{2}-1\right) \frac{d x}{d t}+x=\beta \lambda \cos \lambda t
$$

We find the range of values of $b$ in $(0,2 / 3)$ for which equation (1.1) may have 
3 stable subharmonic solutions. We then, integrate equation (1.1) numerically and a comparison of the numerical results obtained will be compared with the analytical results of this paper.

\section{Matched Local Asymptotic Solutions}

4.1 Outline of the Method. We use the method of matched asymptotic expansions, i.e., expansions which are valid asymptotically in different domains but proportional in domains of overlap.

This method of asymptotic expansions is related to Cole and Kevorkian's method [5] for solving the autonomous Van der Pol's equation. The method of matched expansions yields formal local asymptotic solutions in which the constants of integrations determined by avergaging and by matching pairs of local solutions of adjacent regions. The trajectory of periodic solution of equations (1.1) with $k$ large (i.e. $k>k_{0}(b, \lambda)$ ) have a characteristic behaviour. We divide the trajectory over half of its period to two main regions $R_{1} R_{2}$, and a third narrow strip $R_{3}$ (Fig. 1). In the region $R_{1}$ the solution decays from the value 2 to 1 and has a small amplitude oscillations of period $2 \pi / \lambda$ (lemma 1 ). In the region $R_{2} 1-A k^{\frac{-1}{2}} \leq x \leq 1+A k^{\frac{-1}{2}}$. In the third region $R_{3}$ the trajectory jumps from 1 to 2 in a time $o(1)$ and the trajectory in this region crosses the $t$-axis almost vertically as in Fig. 1.

4.2 The Expansion for the Region $R_{1}$. In this region we apply a two variable expansions procedure. To do so we introduce a second independent variable,

$$
\Theta=\left(t-t_{0}\right) k^{-1}
$$

and suppose that the solution can be written as

$$
x(t, \Theta)=x_{0}(t, \Theta)+x_{1}(t, \Theta) k^{-1}+o\left(k^{-1}\right)
$$

Substituting (4.2.2) into (3.1) and then letting $k \rightarrow \infty$, we find the following limit equations

$$
\left(x_{0}^{2}-1\right) \frac{\partial x_{0}}{\partial t}=0
$$


and

$$
\left(x_{0}^{2}-1\right)\left[\frac{\partial x_{1}}{\partial t}+\frac{\partial x_{0}}{\partial \Theta}\right]+x_{0}=\beta \lambda \cos \lambda t
$$

Equation (4.2.3) implies that $x_{0}$ is a function of $\Theta$ only. Integrating equation (4.2.4) for $x_{1}$, we find

$$
x_{1}(t, \Theta)=\frac{\beta \sin \lambda t}{x_{0}^{2}-1}+\frac{t}{1-x_{0}^{2}}\left[\left(x_{0}^{2}-1\right) \frac{\partial x_{0}}{\partial \Theta}+x_{0}\right]+C_{1}(\Theta),
$$

where $C_{1}(\Theta)$ is the constant of integration. The second term in the right hand side of (4.2.5) is secular in the $t$ variable, so we have to set this term equal zero,

$$
\left(x_{0}^{2}-1\right) \frac{\partial x_{0}}{\partial \Theta}+x_{0}=0 .
$$

Integrating equation (4.2.6) for $x_{0}$, we find

$$
\ln \left|x_{0}\right|-1 / 2\left(x_{0}^{2}-1\right)=\Theta+C_{0}
$$

where $C_{0}$ is the constant of integration. Without loss of generality the constant $C_{0}$ can be taken zero; the constant $t_{0}$ in (4.2.1) already accounts for this contribution, since $C_{0}$ is, in fact, denotes a shift in $\Theta$. A higher order approximation shows that $C_{1}(\Theta)$ can also be taken zero since $C_{1}(\Theta)$ can be written as $C_{1}(\Theta)=C_{0} \psi(\Theta)$. Thus

$$
x_{1}(t, \Theta)=\frac{\beta \sin \lambda t}{x_{0}^{2}(\Theta)-1}
$$

As $t$ approaches $t_{0}$ from the left, the behaviour of $x_{0}$ and $x_{1}$ is

$$
x_{0} \simeq 1+\left(t_{0}-t\right)^{\frac{1}{2}} k^{-\frac{1}{2}}, x_{1} \simeq \frac{\beta \sin \lambda t}{2 \sqrt{t_{0}-t}} k^{\frac{1}{2}},
$$

and therefore the constructed solution (4.2.2) is

$$
x \simeq 1+\left(t_{0}-t\right)^{\frac{1}{2}} k^{-\frac{1}{2}}+\left(\frac{\beta}{2} \sin \lambda t\right)\left(t_{0}-t\right)^{-\frac{1}{2}} k^{-\frac{1}{2}}+o\left(k^{-1}\right)
$$

This solution behaves singular and looses its validity as $t \rightarrow t_{0}$ from the left. At the beginning of the region $R_{1}(x, t)=\left(2, t_{-1}^{-}\right)$. Expand equation (4.2.7) about $x_{0}=2$ to obtain

$$
x_{0}(\Theta)=2-2 / 3(\Theta-\ln 2+3 / 2)+\cdots
$$


Since as $x_{0} \rightarrow 2 x_{1} \rightarrow \frac{\beta \sin \lambda t_{-1}^{-}}{3}$, we get from equation (4.2.10) and (4.2.11) that

$$
t_{-1}^{-}=t_{0}-(3 / 2-\ln 2) k+\frac{\beta}{2} \sin \lambda t_{-1}^{-} \text {. }
$$

4.3 The Expansion for the Region $R_{2}$. In this region $t=t_{0}+2 m \pi / \lambda$ and $1-A k^{\frac{-1}{2}} \leq x \leq 1+A k^{\frac{-1}{2}}$. we suppose the solution can be written as

$$
x=1+g(t) k^{\frac{-1}{2}}+o\left(k^{\frac{-1}{2}}\right)
$$

Substituting (4.3.1) into equation (3.1) and letting $k \rightarrow \infty$ we obtain

$$
2 g \frac{d g}{d t}+1=\beta \lambda \cos \lambda t
$$

or

$$
g(t)=\left(\beta \sin \lambda t+t_{0}-t+C_{2}\right)^{\frac{1}{2}},
$$

where $C_{2}$ is the constant of integration. The function $g(t)$ in (4.3.3) can be expanded as

$$
g(t)=\left(t_{0}-t\right)^{\frac{1}{2}}\left[1+\frac{\beta \sin \lambda t+C_{2}}{2\left(t_{0}-t\right)^{\frac{1}{2}}}+\cdots\right]
$$

For $\left|t_{0}-t\right| \gg 1$, the expansion (4.3.4) behaves as

$$
g(t) \simeq\left(t_{0}-t\right)^{\frac{1}{2}}+\left[\frac{\beta}{2} \sin \lambda t+C_{2}\right]\left(t_{0}-t\right)^{\frac{-1}{2}}
$$

so that the constructed solution (4.3.1) is

$$
x \simeq 1+\left(t_{0}-t\right)^{\frac{1}{2}} k^{\frac{-1}{2}}+\left[\frac{\beta}{2} \sin \lambda t+C_{2}\right]\left(t_{0}-t\right)^{\frac{-1}{2}} k^{\frac{-1}{2}}+o\left(k^{\frac{-1}{2}}\right)
$$

The solution (4.3.6) matches the solution (4.2.10) if $C_{2}=0$. The solution (4.3.6) intersects the line $x=1$ at $t=t_{0}^{-}$where $t_{0}^{-}$is the solution of the equation $g\left(t_{0}^{-}\right)+o\left(k^{\frac{-1}{2}}\right)=0$. With an accuracy of $o\left(k^{\frac{-1}{2}}\right) t_{0}^{-}$is the solution of the equation

$$
t_{0}^{-}=t_{0}-\beta \sin \lambda t_{0}^{-}
$$


4.4 The Expansion for the Region $\bar{R}_{1}$. Since the trajectories of the equation (3.1) are skew-symmetric, the asymptotic expansion for the solution in this region may be written as

$$
x=x_{0}^{-}(\tau)+x_{1}^{-}(t, \tau) k^{-1}+o\left(k^{-1}\right)
$$

where

$$
\tau=\left(t-t_{1}\right) k^{-1}
$$

Then we have

$$
\begin{gathered}
\ln \left(-\bar{x}_{0}\right)-\frac{1}{2}\left(\bar{x}_{0}^{2}-1\right)=\tau, \\
\bar{x}_{1}(t, \tau)=\frac{\beta \sin \lambda t}{\bar{x}_{0}^{2}-1}
\end{gathered}
$$

Since at the beginning of the region $\bar{R}_{1}(x, t)=\left(-2, t_{0}^{-}\right)$, we expand (4.4.3) about $x_{0}^{-}=-2$ to obtain, as in (4.2.12)

$$
t_{1}=t_{0}^{-}-\left(\frac{3}{2}-\ln 2\right) k+\frac{\beta}{2} \sin \lambda t_{0}^{-}+o\left(k^{-1}\right) .
$$

4.5 The Expansion for the Region $\bar{R}_{2}$. Similar to the expansion (4.3.1), we assume the expansion for this region as

$$
x=-1-\bar{g}(t) k^{\frac{-1}{2}}+o\left(k^{\frac{-1}{2}}\right),
$$

where

$$
\bar{g}(t)=\left(-\beta \sin \lambda t+t_{1}-t\right)^{\frac{1}{2}}
$$

The solution (4.5.1) intersects the line $x=1$ at $t=\bar{t}_{1}$ where $\bar{t}_{1}$ satisfies the equation

$$
t_{1}-t_{1}^{-}=\beta \sin \lambda t_{1}^{-}
$$


5.

5.1 Periodicity Conditions. We consider the periodic solution with pe$\operatorname{riod} T=2 m \pi / \lambda$ which intersect the $t$-axis only twice in the period. Such solution satisfies

$$
t_{1}^{-}-t_{-1}^{-}=2 m \pi / \lambda
$$

Let $\Delta(k)$ be defined as $\Delta(k)=T_{0}-t$, where $T_{0}$ as in (1.2). Therefore

$$
\Delta=(3-2 \ln 2) k-2 m \pi \lambda+\Theta\left(k^{\frac{-1}{3}}\right)
$$

The system of equations (4.2.12), (4.3.7), (4.4.5), (4.5.3) and (5.1.1) can be reduced to the system

$$
\begin{gathered}
\sin \left(\lambda t_{1}^{-}\right)-\sin \left(\lambda t_{0}^{-}\right)=\frac{2 \Delta}{3 \beta}, \\
\sin \left(\lambda t_{1}^{-}\right)+\sin \left(\lambda t_{0}^{-}\right)=4 \pi m / \lambda \beta-4\left(t_{1}^{-}-t_{0}^{-}\right),
\end{gathered}
$$

For convenience we introduce the following notations

$$
\begin{aligned}
t_{-1}^{-} & =2 \mu_{-1} \pi+w_{-1} \\
t_{0}^{-} & =\left(2 \mu_{0}+1\right) \pi+w_{0} \\
t_{1}^{-} & =2 \mu_{1} \pi+w_{1}
\end{aligned}
$$

where $-\pi<w_{i} \leq \pi, i=1,0,1$. Since the solutions are periodic, we have $w_{-1}=w_{1}$.

Each of the equation (4.3.7) and (4.5.3) have a unique solution only if $\beta \leq 1$. For $\beta>1$, the equations has more than one solution. We select the smallest root of each equation. To do so we have to set the following condition

$$
w_{i}+\beta \sin \lambda w_{i}>\left(\beta^{2}-1\right)^{\frac{1}{2}}-\arccos (1 / \beta)-\pi / \lambda, \quad i=0,1
$$

5.2 The Case $m$ is Odd. Suppose $m=2 n+1$.

For

$$
2 \mu_{1}-\left(2 \mu_{0}+1\right)=\left(2 \mu_{0}+1\right)-2 \mu_{-1}=m=2 n+1,
$$




$$
w_{0}=w_{1}
$$

Equation (5.1.3) is satisfied. Substituting in (5.1.4) gives

$$
w_{0}=w_{1}=\arcsin \left(\frac{\triangle}{3 \beta}\right)
$$

Therefore another restriction of the parameters is

$$
|\triangle| \leq 3 \beta
$$

Hence the conditions (5.1.6) becomes

$$
\arcsin \left(\frac{\triangle}{3 \beta}\right)+\frac{2 \beta}{3}>\left(\beta^{2}-1\right)^{\frac{1}{2}}-\arccos \left(\frac{1}{\beta}\right)-\frac{\pi}{\lambda}
$$

5.3 The Case $m$ is Even. Suppose $m=2 n$, and set

$$
\begin{aligned}
2 \mu_{1}-\left(2 \mu_{0}+1\right) & =2 n-1, \\
\left(2 \mu_{0}+1\right)-2 \mu_{-1} & =2 n+1
\end{aligned}
$$

In this case the equations (5.1.3) and (5.1.4) does not admit a solution of the type $w_{0}=w_{1}$. We set $\bar{w}_{1}=w_{1}+\pi$ and applying the mean value theorem

$$
\frac{\sin ^{2} \lambda w_{0}-\sin ^{2} \lambda \bar{w}}{w_{0}-\bar{w}_{1}}=\frac{8 \Delta}{3 \beta^{2}}
$$

The solvability condition is

$$
|8 \Delta| \leq 3 \beta^{2}
$$

In Fig. 2 we give the regions where a numerical solutions for (5.1.3) and (5.1.4) was found that satisfied (5.2.4), (5.2.5) and (5.3.3).

6. Conclusion and Comparisons with Numerical and Analytical Results

For given fixed value of $k$ say $k=k_{0}$, the restrictions (5.2.4) and (5.3.3) can be read as

$$
\frac{\triangle}{3 k_{0}} \leq b<\frac{2}{3},
$$




$$
\frac{1}{k_{0}}\left(\frac{8 \triangle}{3}\right)^{\frac{1}{2}} \leq b<2 / 3
$$

respectively.

The regions in the $b, k$-plane corresponding with subharmonics of period $(2 n+1) 2 \pi / \lambda$ and $(2 n-1) 2 \pi / \lambda$ overlap. For a value of $b$ and $k$ in the domain of over lap two different solutions are possible depending on the initial values. The region corresponding with a subharmonic of even order overlaps the two regions mentioned above in such a way that in a very narrow strip three subharmonics might exist. we have integrated equation (1.1) numerically. A series of computer simulations has been made to compare the results of this formal asymptotic method with numerical solutions of (1.1) for different values $b, k$ and $\lambda$. In some cases, two periodic solutions with different periods are found depending on the initial values. These are normal subharmonics of odd order. Table 1 listed some of numerical results obtained. These solutions are skew symmetrics (c.f. Fig. $3)$. For $b$ small one may also expect periodic solutions with period $4 \pi n$; these are nonsymmetric solutions (see Fig. 4). Table 2 listed the even subharmonics obtained. Unfortunately we were not lucky enough to find numerically 3 stable subharmonics for the same values of the parameters. 
Table 1.a.

\begin{tabular}{|c|c|c|}
\hline \multicolumn{3}{|r|}{$\lambda=1$} \\
\hline$k$ & $b$ & order of the subharmonic \\
\hline 20 & $\begin{array}{c}0<b \leq 0.32 \\
0.33 \leq b \leq 0.37 \\
0.38 \leq b \leq 0.64 \\
0.65 \leq b \leq 0.68 \\
0.68<b\end{array}$ & $\begin{array}{c}5 \\
5,3 \\
3 \\
3,1 \\
1\end{array}$ \\
\hline 30 & $\begin{array}{c}0<b \leq 0.26 \\
0.27 \leq b \leq 0.30 \\
0.31 \leq b \leq 0.43 \\
0.44 \leq b \leq 0.48 \\
0.49 \leq b \leq 0.67 \\
0.68 \leq b \leq 0.69 \\
0.69<b\end{array}$ & $\begin{array}{c}7 \\
7,5 \\
5 \\
5,3 \\
3 \\
3,1 \\
1\end{array}$ \\
\hline
\end{tabular}

Table 1.b.

\begin{tabular}{|c|c|}
\hline$k$ & $T_{0}(k)$ \\
\hline 0.2 & 6.30000 \\
0.6 & 6.4220 \\
1 & 6.6870 \\
2 & 7.6310 \\
3 & 8.8613 \\
4 & 10.2072 \\
5 & 11.6055 \\
6 & 13.0550 \\
8 & 16.0740 \\
10 & 19.1550 \\
20 & 31.7823 \\
30 & 51.8737 \\
\hline
\end{tabular}


Table 2.

\begin{tabular}{|l|l|l|c|}
\hline$k$ & \multicolumn{1}{|c|}{$b$} & $\lambda$ & order \\
\hline 4 & 0.4 & 4 & 6 \\
4 & 0.5 & 4 & 16 \\
6 & $1 / 6$ & 3 & 6 \\
6.1 & 0.25 & 2 & 4 \\
14 & 0.143 & 1 & 4 \\
\hline
\end{tabular}

\section{References}

[1] M. L. Cartwright, "Forced Oscillations in Nonlinear Systems", Contributions to the Theory of Nonlinear Oscillations, Vol. 1. Annals of Math. Studies No. 20.

[2] A. A. Dorodnicyn, "Asymptotic Solution of the Van der Pol Equation", Prikl. Mat. Mekh., 11 (1947), p. 313-328; Am. Math. Soc. Transl., series 1, 4 (1962), p. 1-23.

[3] E. M. Elabbasy, "Periodic Solutions of Nonlinear Differential Equations", a Numerical Investigation. Ph. D. thesis, Univ. of Wales UK, 1980.

[4] E. M. Elabbasy, "On the periodic solution of the Van der Pol oscillator with large damping", Proceedings of the Royal Society of Edinburgh, 100A, 103-106, 1985.

[5] J. Kevorkian and J. D. Cole, "Perturbation methods in Applied Mathematics", Springer Verlag., New York, 1981.

[6] J. E. Littlewood, "On nonlinear differential equations of the second order: III. The equation $\ddot{x}+k\left(x^{2}-1\right) \dot{x}+x=k b \mu \cos (\mu t+\propto)$ for $k$ large and its generalization", Acta. Math. 97, 267-308.

[7] J. E. Littlewood, "Some problems in real and complex analysis", Mass. Healh, 1968.

[8] M. Urabe, "Periodic Solutions of Van der Pol's Equation with Damping Coefficient $k=$ $0 \sim 10 "$, IRE Transactions on Circuit Theory., 1960, p. 382-386.

Department of Mathematics, College of Science, King Saud University, P. O. Box 2455, Riyadh11451, Saudi Arabia.

New Address: Dept. Maths., Faculty of Science, Mansoura Univ., Mansoura, Egypt. 